\title{
Leptophilic Dark Matter and AMS-02 Cosmic-ray Positron Flux
}

\author{
Qing-Hong Cao, $, 2,3, *$ Chuan-Ren Chen, $, 4,+$ and Ti Gong $1,+\oplus$ \\ ${ }^{1}$ Department of Physics and State Key Laboratory of Nuclear Physics and Technology, \\ Peking University, Beijing 100871, China \\ ${ }^{2}$ Collaborative Innovation Center of Quantum Matter, Beijing, China \\ ${ }^{3}$ Center for High Energy Physics, Peking University, Beijing 100871, China \\ ${ }^{4}$ Department of Physics, National Taiwan Normal University, Taipei 116, Taiwan
}

\begin{abstract}
With the measurement of positron flux published recently by AMS-02 collaboration, we show how the leptophilic dark matter fits the observation. We obtain the percentages of different products of dark matter annihilation that can best describe the flux of high energy positrons observed by AMS. We show that dark matter annihilates predominantly into $\tau \tau$ pair, while both $e e$ and $\mu \mu$ final states should be less than 20\%. When gauge boson final states are included, the best branching ratio of needed $\tau \tau$ mode reduces.
\end{abstract}

\footnotetext{
*Electronic address: qinghongcao@pku.edu.cn

$\dagger$ Electronic address: crchen@ntnu.edu.tw

${ }^{\ddagger}$ Electronic address: ttigong@pku.edu.cn
} 


\section{INTRODUCTION}

The existence of dark matter has been firmly supported by many astrophysical and cosmological observations. However, we have very little knowledge about what dark matter is made of and how it interacts with particles in the Standard Model. Currently, there are many experiments searching for dark matter. The so-called indirect search looks for the products of dark matter annihilation in our Universe. It drew lots of attention recently since both PAMELA [1] and AMS-02 [2, 3] experiments observed an anomaly in cosmic-ray positron fraction measurement. An excess of positron in the energy region $E \gtrsim 10 \mathrm{GeV}$ can not be explained by the known sources. It indicates that there exists a source of primary positrons nearby. One possibility for the origin of these high energy positrons is dark matter [4-13].

Furthermore, the flux of positron observed by PAMELA shows that the energy spectrum of positron is harder than background expectation [14, 15] for energy of positron larger than about $30 \mathrm{GeV}$. This behavior is confirmed by the latest AMS-02 result [16]. In addition to cosmic-ray positron data, the antiproton data observed by PAMELA [17] shows that the antiproton flux is consistent with the background, which suggests that the cosmic-ray antiprotons are mainly secondary. Combining cosmic-ray positron and antiproton data, a leptophilic dark matter candidate that annihilates predominately into leptons is attractive since it produces large amount of energetic positrons while the antiproton flux is suppressed. There are several leptophilic dark matter candidates have been studied in the literature [1822]. In this paper, we focus on the leptophilic dark matter scenario in which charged leptons are predominantly produced when dark matter annihilates. With the precision measurements of positron flux by AMS-02 [16], we vary the fraction of individual charged lepton mode $\left(e^{+} e^{-}, \mu^{+} \mu^{-}, \tau^{+} \tau^{-}\right)$and find the best fit for the shape of spectrum. A proper

total annihilation cross section is chosen to reach the minimum $\chi^{2}$. For comparison, we also consider the case that the leptophilic dark matter annihilates into massive gauge bosons $W^{+} W^{-}$or $Z Z$.

The rest of paper is organized as follows. In section [II, we briefly review the propagation of positron and antiproton, and present the relative parameters in our numerical study. The section III is the numerical results showing our fitting with latest AMS-02 result. Finally, we conclude in section IV. 


\section{COSMIC-RAY POSITRON AND ANTIPROTON PROPAGATION}

After being produced in the processes of dark matter annihilation, the stable particles will propagate in the magnetic field of the Milky Way. For positrons, the energy spectrum can be obtained by solving the flowing diffusion equation when one consider only the dominant contributions from spatial diffusion and energy lost [23],

$$
\nabla \cdot\left[K(E, \vec{r}) \nabla f_{e^{+}}\right]+\frac{\partial}{\partial E}\left[b(E, \vec{r}) f_{e^{+}}\right]+Q(E, \vec{r})=0
$$

where $f_{e^{+}}$is the number density of $e^{+}$per unit kinetic energy, $K(E, \vec{r})$ is the diffusion coefficient, $b(E, \vec{r})$ is the rate of energy loss and $Q(E, \vec{r})$ is the source of $e^{+}$. Here the primary positrons are produced in dark matter annihilation,

$$
Q(E, \vec{r})=\frac{1}{2}\left(\frac{\rho(\vec{r})}{m_{D M}}\right)^{2} \sum_{i}\langle\sigma v\rangle_{i}\left(\frac{d N_{e^{+}}}{d E}\right)_{i},
$$

where $\rho(\vec{r})$ is the dark matter profile in the Milky Way, $m_{D M}$ is the mass of dark matter, $\left(d N_{e^{+}} / d E\right)_{i}$ is the energy spectrum of $e^{+}$from dark matter annihilation into any possible final state $i$ that generates electrons, with annihilation cross section $\langle\sigma v\rangle_{i}$. In our study, $i=e^{+} e^{-}, \mu^{+} \mu^{-}, \tau^{+} \tau^{-}, W^{+} W^{-}$or $Z Z$. The flux of $e^{+}$originated from dark matter is then given by

$$
\Phi_{e^{+}}^{D M}(E)=\frac{c}{4 \pi} f_{e^{+}}\left(E, r_{\odot}\right),
$$

where $c$ is the speed of light and $r_{\odot} \sim 8.5 \mathrm{kpc}$ is the distance from the Milky Way center to the Sun. We use micrOMEGAs 4 [24] with its default settings to calculate the propagation of positrons that originate from dark matter annihilation. The Zhao dark matter profile [31] is used.

In addition to $e^{+}$flux from dark matter decay, there exists a secondary $e^{+}$flux from interactions between cosmic rays and nuclei in the interstellar medium. The secondary positron $\Phi_{e^{+}}^{s e c}$ can be well approximated as [25, 26]

$$
\Phi_{e^{+}}^{\sec }(E)=\frac{4.5 E^{0.7}}{1+650 E^{2.3}+1500 E^{4.2}} \quad\left[\mathrm{GeV}^{-1} \mathrm{~cm}^{-2} \mathrm{sec}^{-1} \mathrm{sr}^{-1}\right]
$$

where $E$ is in unit of $\mathrm{GeV}$.

The propagation of antiprotons, neglecting the energy lost, can be described as

$$
K_{p} \nabla^{2} f_{\bar{p}}(T, \vec{r})-V_{c} \frac{\partial}{\partial z} f_{\bar{p}}(T, \vec{r})-2 h \delta(z) \Gamma_{a n n} f_{\bar{p}}(T, \vec{r})+Q(T, \vec{r})=0,
$$


where $f_{\bar{p}}(T, \vec{r})$ is the number density of antiproton per unit energy, $T$ is the kinetic energy of antiproton, $K_{p}$ is the diffusion parameter and $V_{c}$ is related to the convective wind that tends to push antiprotons away from the Galactic plane and is assumed to be a constant. The third term on the left-hand side of Eq. (3) represents the annihilation of $\bar{p}$ with the interstellar proton in the Galactic plane where $h$ is the half-height of plane and $\Gamma_{\text {ann }}$ is the antiproton annihilation rate. The solution of the interstellar flux of antiproton in the vicinity of solar system is [27]

$$
\Phi^{\mathrm{IS}}\left(T, \vec{r}_{\odot}\right)=\frac{v_{\bar{p}}}{4 \pi} f_{\bar{p}}\left(T, \vec{r}_{\odot}\right)
$$

However, when the effects of solar modulation, which are important for the low energy antiprotons, are taken into account, the flux of antiproton obtained at the Earth will be given as [28, 29]

$$
\frac{d \Phi_{\overline{\bar{p}}}^{\odot}}{d T_{\odot}}=\frac{p_{\odot}^{2}}{p_{I S}^{2}} \frac{d}{d T_{I S}} \Phi^{\mathrm{IS}}\left(T_{I S}, \vec{r}_{\odot}\right),
$$

where $T_{\odot}=T_{\mathrm{IS}}-\phi_{F}$ is the kinetic energy of antiproton observed at the Earth with $\phi_{F}$ being the solar modular parameter; $p_{\odot}$ and $p_{I S}$ are the momentum of antiproton at the Earth and in the interstellar medium, respectively. The astrophysical antiproton $\Phi_{\bar{p}}^{b g}$ background can be written as a simple fitting function provided in Ref. [30],

$$
\Phi_{\bar{p}}^{b g}=\frac{0.9 t^{-0.9}}{14+30 t^{-1.85}+0.08 t^{2.3}}\left[\mathrm{GeV}^{-1} \mathrm{~m}^{-2} \mathrm{~s}^{-1} \mathrm{sr}^{-1}\right]
$$

where $t$ is the kinetic energy of $\bar{p}$. Note that all the propagation parameters are chosen as the default settings in micrOMEGAs.

\section{DARK MATTER AND AMS-02 POSITRON FLUX}

Figure 1 displays the positron flux data observed by AMS-02, compared with the secondary positrons from background. We focus only on the high energy positron above $20 \mathrm{GeV}$ such that we do not have to consider the effects of solar modulation that are significant for low energy positrons [16]. The rise of AMS-02 data at $E \sim 30 \mathrm{GeV}$ shows that the spectral index is larger than -3 , and such a behavior certainly can not be explained by the secondary positrons. In the bottom panel of Fig. 1, we plot the shapes of positron flux $\left(E^{3} \Phi_{e^{+}}\right)$for different leptonic final states after propagation. The behavior of cosmic-ray positron varies 


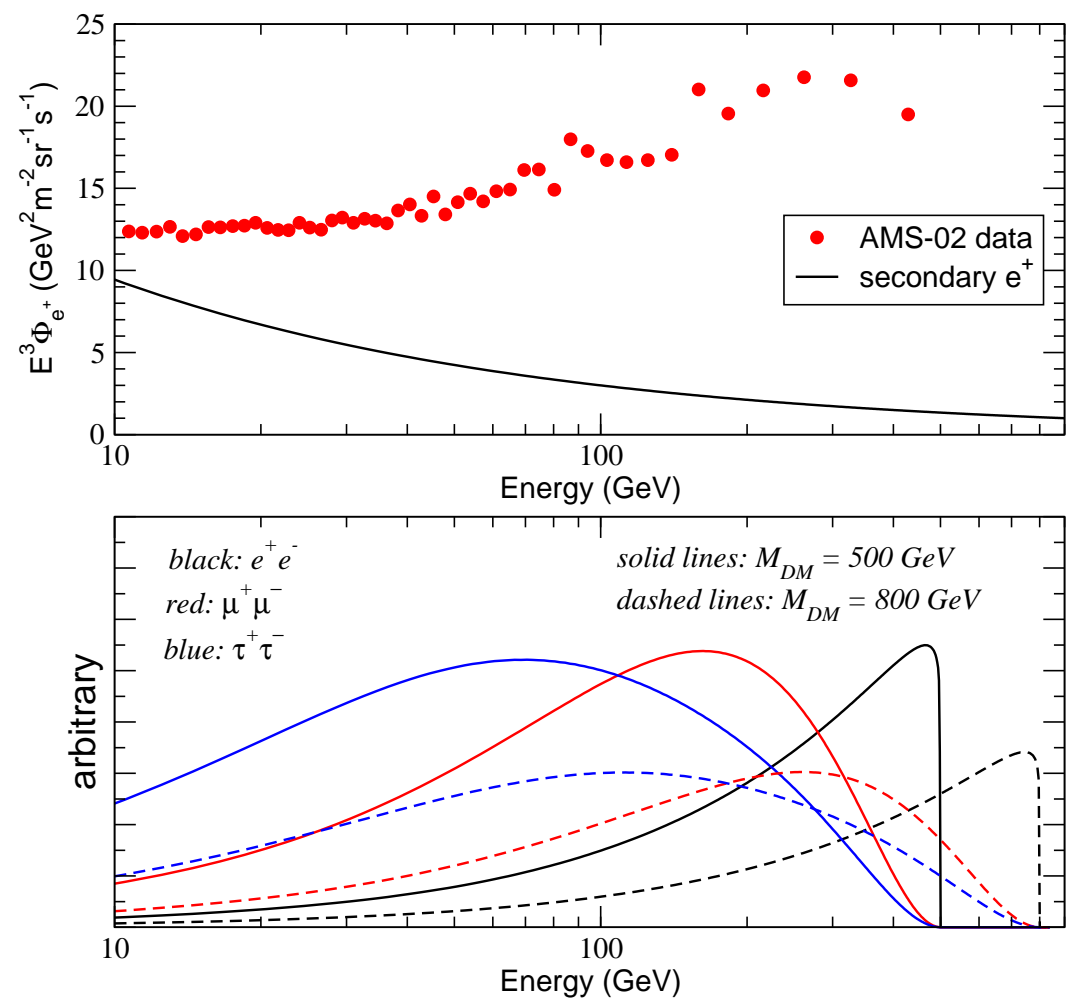

FIG. 1: (Top) The comparison between AMS-02 updated positron flux data and expected secondary positron flux. (Bottom) The comparison of positron fluxes from different final charged leptons in the final state of dark matter annihilation. Note that we multiply different factors to $\mu \mu$ and $\tau \tau$ modes for a better comparison in shape.

significantly for different lepton modes. The ee mode, as originating directly from dark matter annihilation, yields the hardest cosmic-ray positron. The flux peaks at energy around the mass of dark matter and drops quickly. The $\mu \mu$ mode peaks at energy that is close to half of dark matter mass, while the $\tau \tau$ mode reaches the top at energy even lower.

To explain the AMS-02 positron flux with leptophilic dark matter, we adopt a minimal $\chi^{2}$-fit to obtain the best values of the total annihilation cross section as well as the ratios of the leptonic modes from dark matter annihilation for a chosen dark matter mass; see Fig. 2. Among all the AMS-02 data, we consider the positron with energy larger than $20 \mathrm{GeV}$ to safely avoid the effects of solar modulation in our $\chi^{2}$-fit. Figure 2(a) shows the annihilation cross section is around $10^{-23}-10^{-22} \mathrm{~cm}^{3} / \mathrm{s}$, which is about 1000 times larger than the thermal cross section $\langle\sigma v\rangle=3 \times 10^{-26} \mathrm{~cm}^{3} / \mathrm{s}$. We take the mass of dark matter 

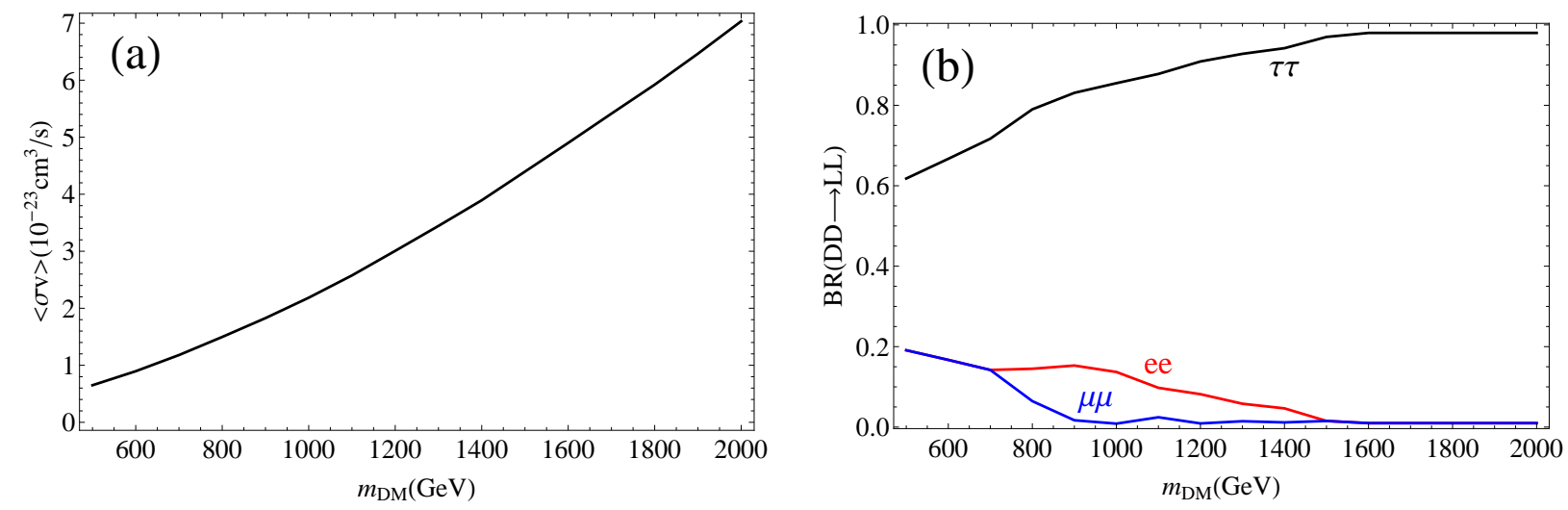

FIG. 2: (a) The annihilation cross section needed for the best fit of AMS-02 updated positron flux data. (b) The fractions of leptonic modes of dark matter annihilation to fit AMS-02 updated data.

candidate to be larger than $500 \mathrm{GeV}$ in order to explain the arising feature of positron energy spectrum. In Fig. 2(b), the $\chi^{2}$-fit shows that the ee-mode should be less than $20 \%$ for a large range of dark matter mass. The $\mu \mu$ mode is also disfavored as the dark matter mass increases; see the blue curve. The $\tau \tau$ mode tends to be dominant for a heavy dark matter candidate. Our fit shows that the three leptonic modes are more or less democratic for a light dark matter around $500 \mathrm{GeV}$. For a dark matter heavier than $1 \mathrm{TeV}$, the $\tau \tau$ mode is well above the other two modes. The dark matter model should consist of a non-trial flavor structure, e.g. the Higgs portal model. In the Higgs portal models the dark matter candidate could predominately decay into vector boson pairs. For comparison, we add in the contributions of gauge boson modes, $W^{+} W^{-}$and $Z Z$, to see how the fitting can be improved.

Figure 3 displays the result of our best values of lepton plus gauge boson modes. The needed annihilation cross section is about the same as the case of purely leptonic final states. However, the fraction of individual mode is changed as $m_{D M}$ is larger than about $600 \mathrm{GeV}$. While the $\tau \tau$ mode is still dominant, but it does not exceed $80 \%$. The $W W$ mode plays a significant role for a heavier dark matter and reaches about $10 \%$ when $m_{D M} \gtrsim 1.4 \mathrm{TeV}$. We notice that the $Z Z$ mode is not important in the fitting. In Fig. 4, we show the flux of positron predicted by the dark matter annihilation using the best values of annihilation cross section and fractions of different annihilation products. The AMS-02 latest data (shown as the blue points with error bars) is also plotted for comparison. It is obvious that the dark 

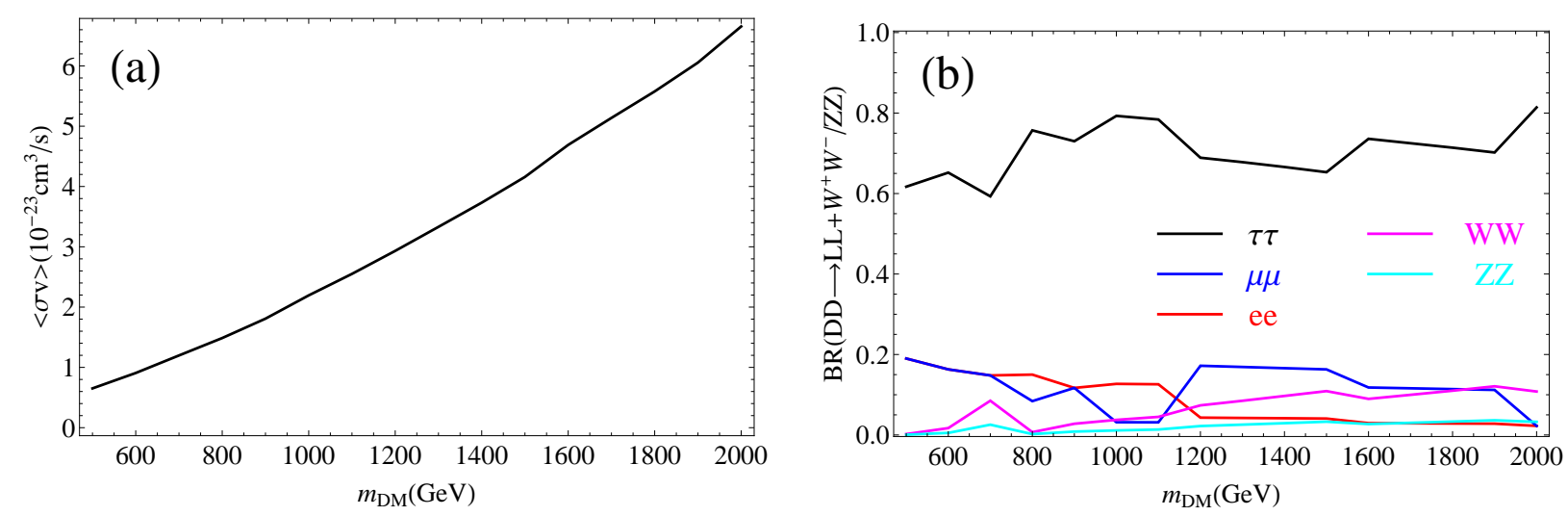

FIG. 3: (a) The annihilation cross section needed for the best fit of AMS-02 positron flux data, including $W W$ and $Z Z$ gauge boson modes. (b) The fractions of different final states of dark matter annihilation to fit AMS-02 positron flux data.
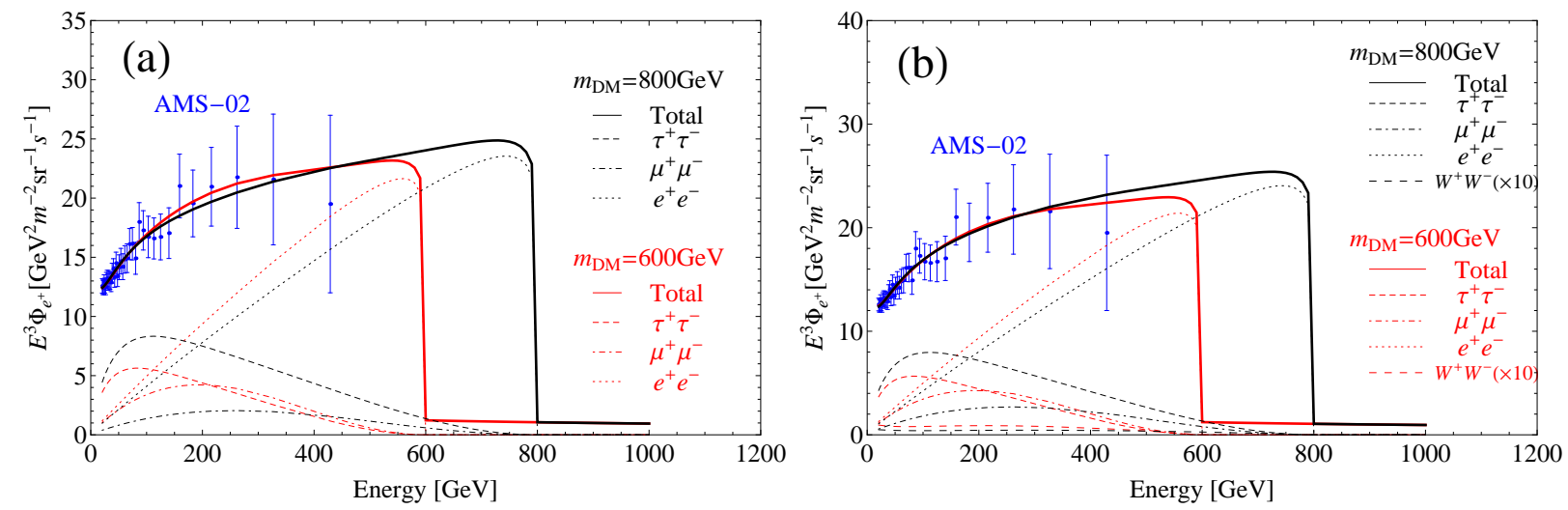

FIG. 4: (a) Comparison between AMS-02 positron flux data [16] and prediction from dark matter annihilation with best fit of annihilation cross section and fractions of lepton final states. (b) Same as Left, but including $W W$ and $Z Z$ gauge boson modes.

matter could explain the observation well. Here, we only present two benchmark dark matter masses, $600 \mathrm{GeV}$ and $800 \mathrm{GeV}$, for reference. The flux is relatively flat for very high energy positrons and then decreases to pure background prediction at the energy equal to the mass of dark matter. Therefore, the energy where the excess of positron flux vanishes will give us information about the mass of dark matter.

Including the $W W$ mode improved the $\chi^{2}$ fit in general. Among the dark matter masses 


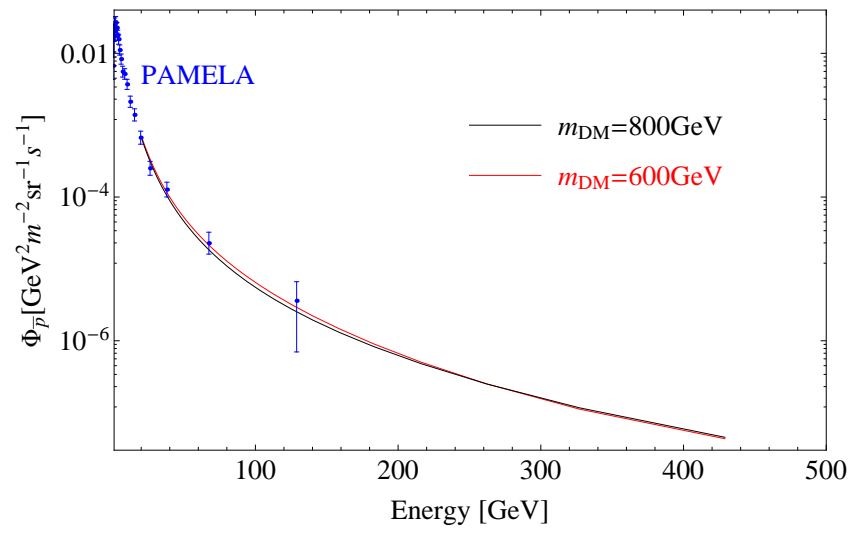

FIG. 5: The antiproton flux generated by the $W W / Z Z$ modes from dark matter annihilation. The blue points represent the PAMELA data [17].

we study, the overall best $\chi^{2}$ is obtained when $m_{D M}=600 \mathrm{GeV}$ for both pure lepton case and the situation that the $W W$ and $Z Z$ modes are considered. However, the $W W$ final state not only generates positrons, but also produces antiprotons that have been measured to be consistent with the background that mainly contains secondary antiprotons [17, 32 -36]. We show in Fig. 5 the flux of antiproton predicted by the dark matter annihilation into the $W W$ final state, with the latest PAMELA observation [17]. Antiproton flux from dark matter annihilation agrees with observation quite well for $600 \mathrm{GeV}$ and $800 \mathrm{GeV}$ dark matter.

\section{DISCUSSION AND CONCLUSIONS}

The AMS-02 collaboration recently published the results of cosmic-ray positrons. The measurement of positron flux for the energy up to $500 \mathrm{GeV}$, with good energy resolution and small uncertainty, allows us to gain more information about dark matter, if we assume dark matter is responsible to the disagreement between data and the known background. In this paper, we study how the leptophilic dark matter can provide explanation of the AMS-02 positron flux data. Our results show that the leptophilic dark matter prefers to annihilate into $\tau \tau$ final state. Both the $e e$ and $\mu \mu$ modes should be less than $20 \%$, and the $\tau \tau$ mode always dominants. The percentage of the $\tau \tau$ mode could reach almost $100 \%$ for the dark matter heavier than about $1.5 \mathrm{TeV}$ unless the $W W$ final state is available. We learn that the $W W$ mode plays a significant role for dark matter heavier than about $1.1 \mathrm{TeV}$. The 
existence of $W W$ final state also brings up the importance of the $\mu \mu$ mode. Combination of the $\mu \mu$ and $W W$ modes could contribute about $30 \%$ of total annihilation cross section, while the ee and $Z Z$ modes can be neglected for $m_{D M} \gtrsim 1.2 \mathrm{TeV}$. We also calculate the antiproton flux generated from the $W W$ final state, and the result is consistent with current PAMELA data.

Finally, we are aware that there is a tension between the $\tau \tau$ final state and cosmic $\gamma$-ray data [37, 38]. However, as we have shown that adding the $W W$ mode in the leptophilic dark matter annihilation reduces the needed fraction of the $\tau \tau$ mode for the best fit. Furthermore, although the result is not shown, we realize that the $\tau \tau$ contribution can be further reduced when quark final states are included. Surely, with contributions of the $W W$ and quark modes, additional antiproton flux will be produced. Therefore, the future antiproton results from AMS-02 are highly expected to tell us more properties of dark matter.

\section{Acknowledgments}

The work of QHC and TG is supported in part by the National Science Foundation of China under Grand No. 11275009. The work of CRC is supported in part by the National Science Council under Grant No. NSC 102-2112-M-003-001-MY3.

[1] O. Adriani et al. [PAMELA Collaboration], Nature 458, 607 (2009) arXiv:0810.4995 [astro$\mathrm{ph}]]$.

[2] M. Aguilar et al. [AMS Collaboration], Phys. Rev. Lett. 110, 141102 (2013).

[3] L. Accardo [AMS Collaboration], Phys. Rev. Lett. 113, 121101 (2014).

[4] M. Cirelli, M. Kadastik, M. Raidal and A. Strumia, Nucl. Phys. B 813, 1 (2009) [Addendumibid. B 873, 530 (2013)] arXiv:0809.2409 [hep-ph]].

[5] Q. Yuan, X. J. Bi, G. M. Chen, Y. Q. Guo, S. J. Lin and X. Zhang, Astropart. Phys. 60, 1 (2015) arXiv:1304.1482 [astro-ph.HE]].

[6] M. Ibe, S. Iwamoto, S. Matsumoto, T. Moroi and N. Yokozaki, JHEP 1308, 029 (2013) arXiv:1304.1483 [hep-ph]].

[7] H. B. Jin, Y. L. Wu and Y. F. Zhou, JCAP 1311, 026 (2013) arXiv:1304.1997 [hep-ph]]. 
[8] M. Ibe, S. Matsumoto, S. Shirai and T. T. Yanagida, JHEP 1307, 063 (2013) arXiv:1305.0084 [hep-ph]].

[9] P. S. B. Dev, D. K. Ghosh, N. Okada and I. Saha, Phys. Rev. D 89 (2014) 095001 arXiv:1307.6204 [hep-ph]].

[10] A. Ibarra, A. S. Lamperstorfer and J. Silk, Phys. Rev. D 89, 063539 (2014) arXiv:1309.2570 [hep-ph]].

[11] Q. Yuan and X. J. Bi, arXiv:1408.2424 [astro-ph.HE].

[12] S. J. Lin, Q. Yuan and X. J. Bi, arXiv:1409.6248 [astro-ph.HE].

[13] M. Ibe, S. Matsumoto, S. Shirai and T. T. Yanagida, arXiv:1409.6920 [hep-ph].

[14] O. Adriani et al. [PAMELA Collaboration], Phys. Rev. Lett. 111, no. 8, 081102 (2013) arXiv:1308.0133 [astro-ph.HE]].

[15] T. Delahaye, J. Lavalle, R. Lineros, F. Donato and N. Fornengo, Astron. Astrophys. 524 (2010) A51 arXiv:1002.1910 [astro-ph.HE]].

[16] M. Aguilar [AMS Collaboration], Phys. Rev. Lett. 113, 121102 (2014).

[17] O. Adriani et al. [PAMELA Collaboration], Phys. Rev. Lett. 105, 121101 (2010) arXiv:1007.0821 [astro-ph.HE]].

[18] C. R. Chen and F. Takahashi, JCAP 0902, 004 (2009) arXiv:0810.4110 [hep-ph]].

[19] P. f. Yin, Q. Yuan, J. Liu, J. Zhang, X. j. Bi and S. h. Zhu, Phys. Rev. D 79, 023512 (2009) arXiv:0811.0176 [hep-ph]].

[20] P. J. Fox and E. Poppitz, Phys. Rev. D 79, 083528 (2009) arXiv:0811.0399 [hep-ph]].

[21] X. J. Bi, P. H. Gu, T. Li and X. Zhang, JHEP 0904, 103 (2009) arXiv:0901.0176 [hep-ph]].

[22] Q. H. Cao, E. Ma and G. Shaughnessy, Phys. Lett. B 673, 152 (2009) arXiv:0901.1334 [hep$\mathrm{ph}]]$.

[23] A. Ibarra and D. Tran, JCAP 0807, 002 (2008) [arXiv:0804.4596 [astro-ph]].

[24] G. Belanger, F. Boudjema, A. Pukhov and A. Semenov, arXiv:1407.6129 [hep-ph].

[25] I. V. Moskalenko and A. W. Strong, Astrophys. J. 493, 694 (1998) astro-ph/9710124.

[26] E. A. Baltz and J. Edsjo, Phys. Rev. D 59, 023511 (1998) astro-ph/9808243.

[27] M. Cirelli, R. Franceschini and A. Strumia, Nucl. Phys. B 800, 204 (2008) arXiv:0802.3378 [hep-ph]].

[28] L.J. Gleeson and W. I. Axford, Astrophys. J. 149, L115 (1967); Astrophys. J. 154, 1011 (1968) 
[29] J. S. Perko, Astron. Astrophys. J. 184, 119 (1987)

[30] E. Nezri, M. H. G. Tytgat and G. Vertongen, arXiv:0901.2556 [hep-ph].

[31] H. Zhao, Mon. Not. Roy. Astron. Soc. 278, 488 (1996) astro-ph/9509122.

[32] S.Orito et al. [BESS Collaboration], Phys. Rev. Lett. 84, 1078 (2000) arXiv:astro-ph/9906426].

[33] K.Abe et al., Phys. Lett. B 670, 103 (2008) arXiv:0805.1754 [astro-ph]].

[34] Y.Asaoka et al., Phys. Rev. Lett. 88, 051101 (2002) arXiv:astro-ph/0109007.

[35] M.Boezio et al. [WiZard/CAPRICE Collaboration], Astrophys. J. 561, 787 (2001) arXiv:astro-ph/0103513.

[36] O. Adriani, G. C. Barbarino, G. A. Bazilevskaya, R. Bellotti, M. Boezio, E. A. Bogomolov, L. Bonechi and M. Bongi et al., Phys. Rev. Lett. 102, 051101 (2009) arXiv:0810.4994 [astro$\mathrm{ph}]]$.

[37] M. Ackermann et al. [Fermi-LAT Collaboration], Phys. Rev. Lett. 107, 241302 (2011) arXiv:1108.3546 [astro-ph.HE]]; M. Ackermann et al. [LAT Collaboration], Astrophys. J. 761, 91 (2012) [arXiv:1205.6474 [astro-ph.CO]].

[38] M. Cirelli, P. Panci and P. D. Serpico, Nucl. Phys. B 840, 284 (2010) arXiv:0912.0663 [astroph.CO]]; M. Papucci and A. Strumia, JCAP 1003, 014 (2010) [arXiv:0912.0742 [hep-ph]]. 